\title{
Certified Amplification: An Emerging Scientific Norm and Ethos
}

\author{
Carole J. Lee (i) \\ Department of Philosophy, University of Washington, Seattle, WA, US \\ Email: c3@uw.edu
}

(Received 15 October 2021; revised 08 January 2022; accepted 21 April 2022; first published online 30 May 2022)

\begin{abstract}
Merton envisioned his norms of science at a time when peer-reviewed journals controlled scientific communication. Technologies for sharing and finding content have since divorced the certification and amplification of science, generating systemic vulnerabilities. Certified amplification - a new Mertonian-styled norm —enjoins their recoupling and introduces a taxonomy of strategies adopted by institutions to close the certification-amplification gap, including the proportioning of the one to the other. Examples illustrating each taxonomic type collectively paint a picture of an ethos employing a rich range of certification and amplification techniques and emerging in a decentralized fashion across heterogeneous objects, communication modalities, and institutions.
\end{abstract}

\section{Introduction}

Robert Merton envisioned his norms of science at a time when peer-reviewed journals controlled scientific communication. Now, thanks to technologies for sharing and finding information online, scientists can archive and establish priority for their research on preprint platforms and amplify their work on blogs and social networks without gatekeeping, creating new dynamics for capturing visibility and annexing broader audiences. With these radical disruptions to science's communication structures, what happens to its normative structure and processes for self-governance?

In this paper, I propose certified amplification as a new Mertonian-styled norm and ethos. First, I argue that, under Merton's framework, the norms should be understood as serving an instrumental role towards the extension of certified knowledge. This meta-normative perspective opens up the possibility that, had the institutional circumstances and challenges been different, science's norms would have needed to be different as well. I will then argue that current technologies generate new systemic vulnerabilities for the extension of certified knowledge due to the divorcing of certification and amplification. Certified amplification - a norm enjoining their recouplingaddresses this problem. I will articulate a taxonomy of different strategies institutions 
have adopted to close the certification-amplification gap. The examples used to illustrate each taxonomic type collectively paint a picture of an ethos employing a rich range of certification and amplification techniques and emerging in a decentralized fashion across a heterogeneous range of objects, communication modalities, and institutional contexts.

\section{A Mertonian meta-norm}

Merton's norms of science are widely endorsed by scientists (Anderson, Martinson, and De Vries 2007) and are thought to express a kind of "moral consensus" (Merton 1973, 169). Disinterestedness is a "distinctive pattern of institutional control" under which scientists systematically scrutinize each others' claims (Merton 1973, 273). Organized skepticism calls for scrutinizing beliefs without the influence of religious, economic, and political institutions (Merton 1973, 277). Universalism requires evaluating claims according to "preestablished impersonal criteria" rather than on "the personal or social attributes of their protagonist" (Merton 1973, 270). And communism - a norm Harriet Zuckerman and Merton took to be technologically and socially institutionalized by the first peer-reviewed journal- "prescribes the open communication of findings to other scientists" (Zuckerman and Merton 1971, 69).

However, for Merton, the norms also "possess a methodologic rationale" (Merton 1973, 270). The norms are "procedurally efficient" means for achieving science's ultimate "institutional goal"-namely, "the extension of certified knowledge" (Merton 1973, 270; italics mine), that is, knowledge claims which are both "socially shared and socially validated" (Merton 1968, 59). This instrumental logic can be seen in Merton's characterization of the norms as bulwarks against perceived threats to scientific progress. Disinterestedness prevents "individuals from profiting through spurious claims, thereby decreasing the rate of fraud found among scientists compared to other professionals" (Merton 1973, 277). Organized skepticism protects the evaluation of scientific claims against resistance from organized religion as well as social and political groups (Merton 1973, 278). Universalism "ensures that individual contributions abiding by the technical norms of empirical evidence and logical consistency are recognized for their advancement of science" without social, political, or nationalistic bias (Merton 1973, 277). And communism "stands in contrast to the privatization of knowledge in a capitalistic economy that would prevent a shared and more efficiently developed body of knowledge" (Merton 1973, 277). These norms of science appear to earn their status by conforming to a meta-norm: that is, that they are instrumental towards the extension of certified knowledge.

Abstracting to this meta-normative level is critical because it allows us to understand that-had the institutional circumstances and challenges been differentscience's norms would have needed to be different as well. The contingency of Merton's norms can be appreciated by contextualizing them historically: when he introduced them in the 1940s, "totalitarian states seemed to threaten both democracy and science" (Csiszar 2020, 11). We can see this preoccupation throughout. Merton contrasts the norm of universalism against examples of intellectually dishonest, nationalistic acts by scientists during World War I. His discussion of disinterestedness censures "presumably scientific pronouncements of totalitarian spokesmen on race or economy or history" (Merton 1973, 277). Organized skepticism protects science 
from the "anti-rationalism" of "modern totalitarian society" (Merton 1973, 278). And communism weighs in on the conflict between socialist versus capitalist systems for sharing and rewarding intellectual property.

If the norms of science are to be instrumental towards the extension of knowledge, then they need to be continually reevaluated and reimagined in the face of new social, political, economic, technological, and legal challenges to scientific progress. Like Merton, we face a time where "attacks upon the integrity of science have led scientists to recognize their dependence on particular types of social structure" (Merton 1973, 267). The influences we worry about, however, are shaped by technologies and institutions with newly configured affordances, dynamics, incentives, and dangers, cleaving the contours for a new norm.

\section{The contemporary decoupling of certification and amplification}

Under Zuckerman and Merton's narrative, ${ }^{1}$ the introduction of peer-reviewed journal publication gave rise to a beneficial system whereby members of the Royal Society-in their triple-roles as reviewers, authors, and readers of the Philosophical Transactionsprotected the quality of published work as a means for burnishing authors' individual reputations, the journal's imprimatur, and the Society's status (Zuckerman and Merton 1971). However, scientific communication and amplification have since evolved in ways that radically decouple certification, public disclosure, and amplification.

Although the preprint platform arXiv has been available since the early 1990s, preprint platforms (and their submissions) have proliferated over the last decade. Most preprint platforms are owned by non-profit academic groups or organizations (e.g., bioRxiv, medRxiv, the suite of "Rxiv" products using infrastructure provided by the Center for Open Science) or by for-profit publishers (e.g., Springer Nature, Elsevier, Wiley) (Kirkham et al. 2020). Preprints allow scientists to claim priority without the delays associated with peer-reviewed publication, archive content in searchable ways, and are often citable in downstream articles and grant applications via assigned Digital Object Identifiers (DOIs) (Kirkham et al. 2020). ${ }^{2}$

A number of innovations in this space are moving towards permitting the certification of preprints either through: an internally structured peer review process with its own community of peer reviewers (Peer Community In); an external peer review service that displays reviews alongside original articles (e.g., PREreview); direct transfer to a journal that will conduct its own peer review (e.g., from bioRxiv or medRxiv to partnering journals); or a publisher's en suite service where in-house preprint submission serves simultaneously as journal submission (e.g., F1000, In Review for select Springer Nature journals). However, in all of these cases, dissemination precedes certification, marking a reversal of the institutional processes described in Zuckerman and Merton's normative model of science. ${ }^{3}$

\footnotetext{
${ }^{1}$ Historical work has since suggested that routinized expert peer review began at the Transactions more than 150 years after its inception (Moxham and Fyfe 2018).

${ }^{2} \mathrm{~A}$ DOI is a unique, unchanging alphanumeric string assigned to online content, making it easier to identify and retrieve for citations in manuscripts and social media mentions.

${ }^{3}$ An exception is Review Commons, which can deposit refereed preprints to bioRxiv (and submit to affiliate journals).
} 
The gap between the amplification of a scientist's claims and the certification processes used to vet them is widening due to the exponentially increasing volume of scientific research. The Philosophical Transactions began as a monthly periodical (Moxham 2015). Since the beginning of the twentieth century, the volume of peer-reviewed journal articles has grown exponentially, with a twofold increase every twelve years (Dong et al. 2017). By 1968, Merton had already reported that scientists were concerned about their work getting lost in "the flood of published scientific research" (59). Merton recognized that "the vastly increased bulk of publication stiffens the competition between papers for" attention and uptake, which he anticipated would increase the "frequency and intensity" of the Matthew Effect (Merton 1968, 59). ${ }^{4}$ Indeed, current research finds that citations have become more centralized than decentralized (Kim et al. 2020), with disparities between social groups increasing rather than decreasing over time (Dworkin et al. 2020; Bertolero et al. 2020). As he predicted, "social mechanisms that curb or facilitate the incorporation of would-be contributions into the domain of science" (Merton $1968,60)$ are leading to increasingly skewed and unjust allocations of influence and recognition.

Cross-platform reinforcement mechanisms further accelerate disparities in reach and impact. For example, papers that are covered by the media (Phillips et al. 1991), mentioned on social media (Yan and Gerstein 2011), and shared as preprints (Fraser et al. 2020) garner more citations; conversely, citation rates likely inform algorithms driving social media news feeds, follow recommendation systems, publishers' article suggestions, and search engine results (e.g., Google Scholar), thereby accentuating existing disparities in visibility (West and Bergstrom 2021).

Finally, the increasing certification-amplification gap creates social and political costs that threaten to obstruct the scientific enterprise. Although Merton's "communication networks of science" (Merton 1968, 56) were conceived as populations of professional scientists, there is an increasing appreciation that "a key element in how science thrives and flourishes" is through successful communication with policy makers and members of the public (National Academies of Sciences, Engineering, and Medicine 2020,16). Social media has blurred boundaries between public and private relationships (boyd 2010) and connected individuals via ties spanning larger network distances (Bak-Coleman et al. 2021), bringing the "scientist-layman relation" to the fore (Merton 1973, 277). While this enables earnest efforts to share expert information to the public, it also engenders the "abuse of expert authority and the creation of pseudo-sciences" (Merton 1973, 277), including unfounded claims propagated by Nobel Prize winners (Boodman 2021) and sensationalist science (Havstad 2021). Highly broadcast and destabilizing misinformation-about, for example, COVID-19 and climate change-pose an existential threat to the very democratic enterprise thought to provide the "institutional context for the fullest measure of [scientific] development" (Merton 1973, 270).

\footnotetext{
${ }^{4}$ In the Matthew Effect, successful scientists disproportionately accrue visibility and credit for their contributions while the less famous accrue disproportionately less.
} 


\section{Certified amplification as emerging norm and ethos}

Merton's claims about the normative structure of science-and especially Zuckerman and his claims about its emergence-were grounded in the institutionalization of peer-reviewed journals. As such, they foregrounded an assemblage in which the direct object for the certification of knowledge was publication. However, now that scientists have the technological and social means for sharing their claims without gatekeeping, public disclosure is a trivial step compared to what should be understood as the proper object of certification: namely, the amplification of science, i.e., the spread of knowledge claims across individuals and their generated content (e.g, papers, citations, retweets, comments, policy statements). ${ }^{5}$

This shift in focus-away from publication to the more general phenomenon of amplification - can be made to accord with Merton's views by appealing to his conceptualization of what it means to make a "contribution to science" (Merton $1968,59)$. "[F]or science to be advanced, it is not enough that fruitful ideas be originated or new experiments developed or new problems formulated or new methods instituted" (Merton 1968, 59). Instead, "[f]or the development of science, only work that is effectively perceived and utilized by other scientists, then and there, matters" (Merton 1968, 59-60). This requires that scientists outcompete others in "the flow of ideas and findings through the communication networks of science" (Merton 1968, 56). Certified knowledge, then, is not simply "socially shared and socially validated" (Merton 1968, 59) - it is also amplified to some degree.

I propose certified amplification as a contemporary, Mertonian-styled norm of science, which addresses the decoupling of certification and amplification by enjoining their recoupling. Certified amplification is related to disinterestedness insofar as it prescribes the "accountability of scientists to their compeers" through "the exacting scrutiny of fellow experts" (Merton 1973, 276) and recognizes that institutions serve a critical role in facilitating this practice. However, rather than enjoin "the exacting scrutiny of fellow experts" tout court (Merton 1973, 276), certified amplification, as a set of practices, centers the ways that degrees and types of certification and amplification can vary as a function of each other. Certified amplification is also related to organized skepticism, insofar as certification rejects totalitarian-style interference in the evaluation of scientific claims. However, by foregrounding the audiences across which amplification takes place, certified amplification provides a lens for understanding the intellectual and political value of certification across different constituents of the scientific enterprise. ${ }^{6}$

To trace certified amplification's emergence as an ethos, I will look to the "prescriptions, proscriptions, preferences, and permissions" expressed in practices and statements of "institutional values" (Merton 1973, 269) since these drive the evaluative and information communication technology choices shaping the affordances and

\footnotetext{
${ }^{5}$ Peer-reviewed journal articles may continue to be seen as a singular mode of communication for some purposes: for example, peer-reviewed journal articles are more likely to be cited than preprints in policy statements about COVID-19 (Yin et al. 2021).

${ }^{6}$ I do not take conceptual overlap between certified amplification and Merton's original norms to discount its plausibility. Merton himself proposed organized skepticism as a core norm despite its being "variously interrelated with the other elements of the scientific ethos" (Merton 1973, 277).
} 
dynamics of certification and amplification at scale. ${ }^{7}$ I will organize exemplars of this ethos around different functional approaches to recoupling certification and amplification. There is variation in amplification's populations (e.g., communication networks of scientists, policy-makers, the public) and modality (e.g., journals, preprint platforms, search engines, article recommendation services, news, social media). There is also diversity in certification's agents (e.g., journals, national and international health organizations, external peer review services, individual scientists through their citation and social media choices), means (e.g., peer review; open data, code, methods, and materials; pre-registration; replication; reproduction), and target objects (e.g., manuscripts, registered reports, ${ }^{8}$ data, individual people, claims broadcast by news and social media). And some acts of amplification can simultaneously serve as acts of certification (e.g., citations, replications, social media comments). However, the common ethic to recouple certification and amplification is made more remarkable for its decentralized surfacing across a heterogeneous range of objects, institutional contexts, and communication modalities.

\section{I Amplification Conditioned on Certification}

This approach gate-keeps amplification. It was mainstreamed by peer-reviewed journal publication but also appears under different guises across the current scientific landscape. DOIs are assigned to peer-reviewed journal articles as well as to preprints recommended by peer review services (e.g., Peer Community In) and curated by overlay journals (e.g., Open Journal of Astrophysics). ${ }^{9}$ Peer Community In Registered Reports (PCI RR) orchestrates peer review for registered reports and for the following study write ups, where successful manuscripts can be published in any of the twenty-two journals that have agreed to accept articles from this service without further review (PCI RR 2021). Science journalists conduct "informal peer review" by contacting domain-experts to evaluate preprints before deciding whether to cover them (Ordway 2020). And the National Library of Medicine launched the National Institutes of Health (NIH) Preprint Pilot, which makes only preprints written by folks previously vetted via NIH intramural hiring or extramural funding publicly searchable and available on PubMed (PubMed Central 2021). As in the case of journal peer review (McNutt 2019; Zuckerman and Merton 1971), amplification conditioned on certification is a binary mechanism - either content gets amplified or not—and can be used to protect the imprimatur of the institution(s) using their platforms to amplify research.

\subsection{Amplification Proportionate to Certification}

In this strategy, the degree to which some content gets amplified depends on the degree to which it has been certified, where certification can be an ongoing process. It is thought that citations and other reputation-and-authority metrics inform black-boxed

\footnotetext{
${ }^{7}$ In contrast, others have inferred updated Mertonian norms from interviews and surveys of scientists (e.g., Anderson et al. 2010).

${ }^{8}$ Registered reports describe experimental plans before study commencement.

${ }^{9}$ Overlay journals curate collections of preprints and typically consist of links to accepted versions of papers hosted on the article's originating preprint platform.
} 
algorithms that drive search engine and article recommendation results (Jensen 2007; West and Bergstrom 2021). Some have proposed systems for communicating research that aggregate reader assessments and prioritize attention by popularity akin to models used by Reddit, Slashdot, and Stack Exchange (Nosek and Bar-Anan 2012; Tennant et al. 2017). The National Academies of Medicine launched a project to identify principles and attributes for identifying credible sources of health information on social media platforms, which —in the future-could be used to amplify content based on credibility cues such as citations, peer reviewed work, conflict of interest disclosures, and credibility attributes (Kington et al. 2021). Social media platforms have made some efforts to fight misinformation by deplatforming individuals and blocking hashtags (e.g., \#VaccinesKill) promoting discredited information (De Vynck 2021). All of these examples involve amplification tools designed and controlled by (or modeled by) private companies, raising challenges related to public-private coordination, oversight, and transparency (Kington et al. 2021).

\subsection{Amplification with Certification Signals}

Other efforts recouple certification with amplification through changes that ensure that content-however it is discovered-simultaneously delivers credibility cues. For example, journals (e.g., eLife) and preprint review services (e.g., Peer Community In, PREreview, Review Commons) publish reviewer comments alongside articles. Journals (McNutt 2019) and preprint platforms (Soderberg, Errington, and Nosek 2020) can publish statements alongside their articles about open science elements (data, materials, methods, code, and pre-registration of analysis plans) (Aalbersberg et al. 2018) as well as replications, reproductions, statistical rigor and checks, and conflict of interest declarations (McNutt 2019). The credibility of individual articles can also be signaled by "forward linking" to later replications that contextualize a result and by having editorial expressions of concern and retractions flagged across "indexing services (e.g., PubMed, Google Scholar, and DOI-registration agencies) and downstream elements, such as citations in derivative work" (Jamieson et al. 2019, 19234). Certification, in this last example, is a continuous process and requires cross-platform coordination (Jamieson et al. 2019). Perhaps in the future, credibility elements attached to articles and other sources of scientific information could inform procedures for amplifying content proportionate to its certification.

\subsection{Certification Proportionate to Amplification}

In this strategy, the degree to which some content gets certified depends on the degree to which it has (or is expected to be) amplified. Some have argued that replication efforts should be focused on studies with high replication value, which can be measured by "the citation impact of a finding and the precision of the existing evidence of the effect" (Nosek, Spies, and Motyl 2012, 622). The World Health Organization created its "Health Feedback" website, in which experts provided evaluations of scientific claims that had received high visibility in the news or on social media (World Health Organization 2021). Likewise, the Johns Hopkins (2019) Novel Coronavirus Research Compendium assesses emerging research on SARS-CoV-2 and COVID-19, prioritizing both "original, high-quality research for public health action"-an example of amplification conditioned on certification-and "papers 
receiving significant attention, regardless of quality"-an example of certification proportionate to amplification (Johns Hopkins Bloomberg School of Public Health 2021). The preprint platforms bioRxiv and medRxiv enhanced their screening processes to reject manuscripts that could fuel conspiracy theories about COVID-19 (Kwon 2020), effectively raising certification standards for content judged to have high potential for amplification. Note that in a regime where certification is made proportionate with amplification, scientific claims expected to have low amplification value would require less scrutiny, which could make more efficient use of limited reviewer time.

This taxonomy of certified amplification types and their exemplars should not be interpreted as being exhaustive. Nor should the taxonomy be taken to imply incompatibility or competition between approaches. A single platform can embrace all of these features at once: e.g., the PLOS family of journals employs pre-publication peer review (amplification conditioned on certification), publishes peer review reports and reader comments (amplification with certification signals), and provides a widget for sharing on social media for further discussion and evaluation (certification proportionate to amplification and amplification proportionate to certification). But given the highly decentralized nature of scientific certification and amplification, it is even more critical for the extension of certified knowledge that these different types of certified amplification take place across platforms: e.g., a paper may get posted as a preprint with credibility-related statements about open data (amplification with certification signals), undergo peer review for journal publication (amplification conditioned on certification), get amplified on social media for the quality of its data (amplification proportionate to certification), and have its study and data more carefully scrutinized as its visibility increases (certification proportionate to amplification).

\section{Conclusion}

I have argued for certified amplification as a Mertonian-styled norm whose emergence as an ethos is exhibited by decentralized institutional efforts to recouple certification and amplification across heterogeneous objects, communication modalities, and institutional contexts. A number of open questions remain about: inequities and biases in whose contributions get amplified and certified by the scientific community (Buchanan et al. 2021; Krieger et al. 2021; Dworkin et al. 2020; Bertolero et al. 2020); who should be in charge of certification and amplification standards and processes and why (McNutt, Córdova, and Allison 2021); how degrees and types of certification and amplification should vary as a function of the social costs of error (Havstad 2021); public-private coordination, oversight, transparency, and regulation of algorithms amplifying content on internet and social media platforms (Kington et al. 2021; West and Bergstrom 2021); the co-opting and weaponization of markers for scientific credibility and certification by legislators (Levy and Johns 2016) and counterpublics (Lee et al. 2021); how to better support publicly-engaged scientific communication (Ordway 2020); incentives generated by different techniques for closing the certification-amplification gap (Nosek, Spies, and Motyl 2012; Teixeira da Silva and Dobránszki 2015; Jamieson et al. 2019; Heesen and Kofi Bright 2020); remaining gaps between certification and amplification 
in practice; and richer conceptions of certification and amplification as well as new/ updated norms to address the aforementioned challenges.

Finally, because institutions and structures are always changing, the normative structure of science and its ethos will continue to evolve. Of particular interest is the recent formation of the Strategic Council for Research Excellence, Integrity, and Trust-a new body within the National Academies of Sciences, Engineering, and Medicine-designed to provide a more centralized means for "anticipating threats to research integrity and streamlining and improving accountability throughout the research enterprise" (McNutt, Córdova, and Allison 2021, 1). Its ambition is to "discuss, originate, and disseminate best practices, request creation of study committees to issue consensus reports on key issues, and form action collaboratives to implement recommendations" (McNutt, Córdova, and Allison 2021, 1). By articulating a collective vision of how institutions can be structured to promote the extension of certified knowledge and organizing its implementation, the Strategic Council is poised to articulate the norms and ethos for tomorrow's science.

Acknowledgments. Many thanks to the institutional structures that made this paper possible during the COVID-19 pandemic, including (but not limited to): the Philosophy of Science Association which delayed the conference for which this was written; my children's school which reopened fully in the fall of 2021; and, my department chair, Andrea Woody, for helping divine time. Many thanks also to Liam Kofi Bright, Kevin Elliott, Sabina Leonelli, Felipe Romero, Patricia Soranno, and Alison Wylie for their insightful comments. Conflict of interest disclosures: I am a Coordinating Committee Member for the Transparency and Openness Promotion Guidelines and served as a judge for ASAPbio's contest "Encouraging Preprint Curation and Review: A Design Sprint."

\section{References}

Aalbersberg, IJsbrand Jan, Tom Appleyard, Sarah Brookhart, Todd Carpenter, Michael Clarke, Stephen Curry, Josh Dahl, et al. 2018. "Making Science Transparent by Default: Introducing the TOP Statement." OSF Preprints, submitted February 15, 2018. https://doi.org/10.31219/osf.io/sm78t.

Anderson, Melissa S., Brian C. Martinson, and Raymond De Vries. 2007. "Normative Dissonance in Science: Results from a National Survey of US Scientists." Journal of Empirical Research on Human Research Ethics $2(4): 3-14$.

Anderson, Melissa S., Emily A. Ronning, Raymond De Vries, and Brian C. Martinson. 2010. "Extending the Mertonian Norms: Scientists' Subscription to Norms of Research." The Journal of Higher Education 81 (3):366-93.

Bak-Coleman, Joseph B., Mark Alfano, Wolfram Barfuss, Carl T. Bergstrom, Miguel A. Centeno, Iain D. Couzin, Jonathan F. Donges, et al. 2021. "Stewardship of Global Collective Behavior." Proceedings of the National Academy of Sciences 118 (27):e2025764118.

Bertolero, Maxwell A., Jordan D. Dworkin, Sophia U. David, Claudia López Lloreda, Pragya Srivastava, Jennifer Stiso, Dale Zhou, et al. 2020. "Racial and Ethnic Imbalance in Neuroscience Reference Lists and Intersections with Gender." BioRxiv, submitted October 12, 2020. https://doi.org/10.1101/2020. 10.12.336230.

Boodman, Eric. 2021. "He's a Stanford Professor and a Nobel Laureate. Critics Say He Was Dangerously Misleading on Covid." Stat, May 24, 2021. https://www.statnews.com/2021/05/24/stanford-professorand-nobel-laureate-critics-say-he-was-dangerously-misleading-on-covid/.

boyd, danah. 2010. "Social Network Sites as Networked Publics: Affordances, Dynamics, and Implications." In Networked Self: Identity, Community, and Culture on Social Network Sites, edited by Zizi Papacharissi, 47-66. New York, NY: Routledge.

Buchanan, NiCole T., Marisol Perez, Mitch Prinstein, and Idia Thurston. 2021. "Upending Racism in Psychological Science: Strategies to Change How Science Is Conducted, Reported, Reviewed, and Disseminated." American Psychologist 76 (7):1097-112. 
Csiszar, Alex. 2020. "Gaming Metrics Before the Game: Citation and the Bureaucratic Virtuoso." In Gaming the Metrics, edited by Mario Biagioli and Alexandra Lippman, 31-42. Cambridge, MA: The MIT Press.

De Vynck, Gerrit. 2021. "YouTube Is Banning Prominent Anti-Vaccine Activists and Blocking All AntiVaccine Content." The Washington Post, September 29, 2021. https://www.washingtonpost.com/ technology/2021/09/29/youtube-ban-joseph-mercola/.

Dong, Yuxiao, Hao Ma, Zhihong Shen, and Kuansan Wang. 2017. "A Century of Science: Globalization of Scientific Collaborations, Citations, and Innovations." In Proceedings of the 23rd ACM SIGKDD International Conference on Knowledge Discovery and Data Mining, 1437-1446.

Dworkin, Jordan D., Kristin A. Linn, Erin G. Teich, Perry Zurn, Russell T. Shinohara, and Danielle S. Bassett. 2020. "The Extent and Drivers of Gender Imbalance in Neuroscience Reference Lists." Nature Neuroscience 23 (8):918-26.

Fraser, Nicholas, Fakhri Momeni, Philipp Mayr, and Isabella Peters. 2020. "The Relationship Between bioRxiv Preprints, Citations and Altmetrics." Quantitative Science Studies 1 (2):618-38.

Havstad, Joyce C. 2021. "Sensationalist Science, Archaic Hominin Genetics, and Amplified Inductive Risk." Canadian Journal of Philosophy. https://doi.org/10.1017/can.2021.15.

Heesen, Remco, and Liam Kofi Bright. 2020. "Is Peer Review a Good Idea?" The British Journal for the Philosophy of Science 72 (3):635-63.

Jamieson, Kathleen Hall, Marcia McNutt, Veronique Kiermer, and Richard Sever. 2019. "Signaling the Trustworthiness of Science." Proceedings of the National Academy of Sciences 116 (39):19231-36.

Jensen, Michael. 2007. "The New Metrics of Scholarly Authority." Chronicle of Higher Education, June 15, 2007. https://www.chronicle.com/article/the-new-metrics-of-scholarly-authority/.

Johns Hopkins Bloomberg School of Public Health. 2021. "Novel Coronavirus Research Compendium." https://ncrc.jhsph.edu.

Kim, Lanu, Jason H. Portenoy, Jevin D. West, and Katherine W. Stovel. 2020. "Scientific Journals Still Matter in the Era of Academic Search Engines and Preprint Archives." Journal of the Association for Information Science and Technology 71 (10):1218-26.

Kington, Raynard S., Stacey Arnesen, Wen-Ying Sylvia Chou, Susan J. Curry, David Lazer, and Antonia M. Villarruel. 2021. "Identifying Credible Sources of Health Information in Social Media: Principles and Attributes." NAM Perspectives. https://doi.org/10.31478/202107a.

Kirkham, Jamie J., Naomi C. Penfold, Fiona Murphy, Isabelle Boutron, John P. Ioannidis, Jessica Polka, and David Moher. 2020. "Systematic Examination of Preprint Platforms for Use in the Medical and Biomedical Sciences Setting." BMJ Open 10 (12):e041849.

Krieger, Nancy, Rhea W. Boyd, Fernando De Maio, and Aletha Maybank. 2021. "Medicine's Privileged Gatekeepers: Producing Harmful Ignorance about Racism and Health." Health Affairs Blog, April 20. doi: $10.1377 / \mathrm{hblog} 20210415.305480$.

Kwon, Diana. 2020. "How Swamped Preprint Servers are Blocking Bad Coronavirus Research." Nature 581 (7807):130-32.

Lamont, Michèle. 2009. How Professors Think. Cambridge, MA: Harvard University Press.

Lee, Crystal, Tanya Yang, Gabrielle D. Inchoco, Graham M. Jones, and Arvind Satyanarayan. 2021. "Viral Visualizations: How Coronavirus Skeptics Use Orthodox Data Practices to Promote Unorthodox Science Online." In Proceedings of the 2021 CHI Conference on Human Factors in Computing Systems, 1-18.

Levy, Karen EC, and David Merritt Johns. 2016. "When Open Data Is a Trojan Horse: The Weaponization of Transparency in Science and Governance." Big Data \& Society 3 (1):1-6.

McNutt, Marcia, France A. Córdova, and David B. Allison. 2021. "The Strategic Council for Research Excellence, Integrity, and Trust." Proceedings of the National Academy of Sciences 118 (41):e2116647118.

McNutt, Marcia K. 2019. "What Is the Role of Journals in Promoting Trustworthy Research?" AGU Fall Meeting Abstracts 2019:U14E03.

Merton, Robert K. 1968. "The Matthew Effect in Science: The Reward and Communication Systems of Science are Considered." Science 159 (3810):56-63.

Merton, Robert K. 1973. "The Normative Structure of Science." In The Sociology of Science: Theoretical and Empirical Investigations, edited by Norman W. Storer, 267-78. Chicago, IL: University of Chicago Press.

Moxham, Noah. 2015. "Fit for Print: Developing an Institutional Model of Scientific Periodical Publishing in England, 1665-ca. 1714." Notes and Records: The Royal Society Journal of the History of Science 69 (3): $241-60$. 
Moxham, Noah, and Aileen Fyfe. 2018. "The Royal Society and the Prehistory of Peer Review, 1665-1965." The Historical Journal 61 (4):863-89.

National Academies of Sciences, Engineering, and Medicine. 2020. The Endless Frontier: The Next 75 Years in Science. Washington, DC: The National Academies Press. https://doi.org/10.17226/25990.

Nosek, Brian A., and Yoav Bar-Anan. 2012. "Scientific Utopia: I. Opening Scientific Communication." Psychological Inquiry 23 (3):217-43.

Nosek, Brian A., Jeffrey R. Spies, and Matt Motyl. 2012. "Scientific Utopia: II. Restructuring Incentives and Practices to Promote Truth Over Publishability." Perspectives on Psychological Science 7 (6):615-31.

Ordway, Denise-Marie. 2020. "Covering Biomedical Research Preprints Amid the Coronavirus: 6 Things to Know.” The Journalist's Resource: Informing the News, April 2, 2020. https://journalistsresource.org/ health/medical-research-preprints-coronavirus/.

Peer Community In Registered Reports. 2021. "List of PCI RR-Friendly Journals." https://rr. peercommunityin.org/about/pci_rr_friendly_journals.

Phillips, David P., Elliot J. Kanter, Bridget Bednarczyk, and Patricia L. Tastad. 1991. "Importance of the Lay Press in the Transmission of Medical Knowledge to the Scientific Community." New England Journal of Medicine 325 (16):1180-83.

PubMed Central. 2021. "NIH Preprint Pilot." https://www.ncbi.nlm.nih.gov/pmc/about/nihpreprints/.

Soderberg, Courtney K., Timothy M. Errington, and Brian A. Nosek. 2020. "Credibility of Preprints: An Interdisciplinary Survey of Researchers." Royal Society Open Science 7 (10):201520.

Teixeira da Silva, Jaime A., and Judit Dobránszki. 2015. "Problems with Traditional Science Publishing and Finding a Wider Niche for Post-Publication Peer Review." Accountability in Research 22 (1):22-40.

Tennant, Jonathan P., Jonathan M. Dugan, Daniel Graziotin, Damien C. Jacques, François Waldner, Daniel Mietchen, Yehia Elkhatib, et al. 2017. "A Multi-Disciplinary Perspective on Emergent and Future Innovations in Peer Review.” F1000Research 6:1151. https://doi.org/10.12688/f1000research. 12037.3.

West, Jevin D., and Carl T. Bergstrom. 2021. "Misinformation in and About Science." Proceedings of the National Academy of Sciences 118 (15):e1912444117.

World Health Organization. 2021. "Health Feedback.” https://healthfeedback.org.

Yan, Koon-Kiu, and Mark Gerstein. 2011. "The Spread of Scientific Information: Insights from the Web Usage Statistics in PLoS Article-Level Metrics." PLoS One 6 (5):e19917.

Yin, Yian, Jian Gao, Benjamin F. Jones, and Dashun Wang. 2021. "Coevolution of Policy and Science During the Pandemic." Science 371 (6525):128-30.

Zuckerman, Harriet, and Robert K. Merton. 1971. "Patterns of Evaluation in Science: Institutionalisation, Structure and Functions of the Referee System." Minerva 9 (1):66-100.

Cite this article: Lee, Carole J. 2022. "Certified Amplification: An Emerging Scientific Norm and Ethos." Philosophy of Science 89 (5):1002-1012. https://doi.org/10.1017/psa.2022.37 